\title{
Étude comparée de la formation des capsules hémocytaires chez Locusta migratoria, Melolontba melolontba et Calliphora erytbrocephala
}

\author{
par M. BREHELIN, D. ZACHARY et J. A. HOFFMANN \\ Laboratoire de Biologie générale, Faculté des Sciences, \\ 12, rue de l'Université, F 67000 Strasbourg
}

Malgré les nombreux travaux de la littérature (Salt, 1970), plusieurs points concernant les réactions d'encapsulement de corps étrangers chez les insectes restent très discutés. Dans le cadre de notre travail sur les fonctions des hémocytes, nous avons abordé l'étude des catégories de cellules sanguines formant les capsules, de leurs transformations au cours de la réaction et de leur action sur l'implant.

Notre étude a été réalisée sur quatre espèces: Locusta migratoria (Orthoptère), Melolontha melolontha (Coléoptère), Clitimnus extradendatus (Chéleutoptère) et Calliphora erythrocephala (Diptère). Trois de ces espèces présentent une image sanguine semblable (plasmatocytes, granulocytes, coagulocytes) (Hoffmann et al., 1968 ; Devanchelle, 1971) tandis que la quatrième espèce (Calliphora) a une image sanguine très différente (plasmatocytes, œnocytoïdes et thrombocytoïdes) (Zachary et Hoffmann, 1973).

Nous avons implanté, dans différentes régions du corps, des fragments stériles de cellophane, de cat-gut, de coton ou de muscle de bœuf.

Les granulocytes typiques, et eux seuls, forment chez les trois premières espèces (l'Orthoptère, le Coléoptère et le phasme) une capsule autour du corps étranger. Après 48 heures, on peut reconnaître deux zones dans ces capsules: une zone périphérique où les hémocytes ne sont en contact que par leurs prolongements et une zone plus interne où les cellules sont étalées et appliquées les unes aux autres.

Des modifications cytologiques donnent à cette formation une cohésion et une certaine rigidité. On note en particulier l'établissement de desmosomes et surtout une 
importante synthèse de microtubules intracytoplasmiques. Chez Locusta, ces microtubules se groupent par paquets de plusieurs dizaines, entourés d'une substance dense aux électrons. Le nombre des inclusions caractéristiques des granulocytes diminue.

Entre les cellules de la zone périphérique, on observe un fin matériel basal qui peut prendre l'aspect de longues membranes sinueuses. Nous n'avons jamais observé de substances présentant une structure périodique.

Chez Calliphora, qui ne possède pas de granulocytes, seuls les thrombocytoïdes et les fragments anucléés auxquels ils donnent naissance dans l'hémolymphe circulante participent à la formation des capsules. Chez cette espèce, nous n'avons jamais observé ni desmosomes, ni paquets de microtubules. Par contre, l'implant est recouvert d'une substance très dense aux électrons, qui semble différente des mélanines.

Quelle que soit la nature du corps implanté, la formation des capsules hémocytaires présente les mêmes modalités. Nous n'avons jamais pu mettre en évidence de dégradation de l'implant, même après plusieurs semaines d'implantation. Dans le cas d'implantation de matériel non stérile, les microorganismes fixés à la surface du corps étranger conservent leurs potentialités de développement après un encapsulement de 10 jours.

\section{Bibliographie}

Devauchelle (G.), 1971. - Etude ultrastructurale des hémocytes du Coléoptère Melolontha melolontha (L.). J. Ultrastruct. Res., 34, 492-516.

Hoffmann (J.-A.), Stoekel (M.-E.), Porte (A.) et Joly (P.), 1968. - Ultrastructure des hémocytes de Locusta migratoria (Orthoptère). C.R. Acad. Sci., D, 266, 503-505.

SALT (G.), 1970. - The cellular defence reactions of Insects. Cambridge University Press, Cambridge, 116 p.

ZACHARY (D.) et HofFmanN (J.-A.), 1973. - The haemocytes of Calliphora erythrocephala (Meig.) Diptera. Z. Zellforsch. mikr. Anat., 141, 55-73. 